\title{
Sorafenib $\mathbf{N}$-oxide Is an Inhibitor of Human Hepatic CYP3A4
}

Sussan Ghassabian, ${ }^{a}$ Tina B. Gillani, ${ }^{\mathrm{a}}$ Tristan Rawling, ${ }^{\mathrm{b}}$ Severine Crettol, ${ }^{\mathrm{a}}$ Pramod C. Nair, ${ }^{\mathrm{c}}$ and Michael Murray, ${ }^{a, *}$

aPharmacogenomics and Drug Development Group, Discipline of Pharmacology, School of Medical Sciences, Sydney Medical School, University of Sydney, NSW 2006, AUSTRALIA, 'bschool of Mathematical and Physical Sciences, Faculty of Science, University of Technology Sydney, Ultimo, New South Wales, 2007, AUSTRALIA, and 'Department of Clinical Pharmacology and Flinders Centre for Innovation in Cancer, College of Medicine and Public Health, Flinders University, Bedford Park, SA 5042, AUSTRALIA

*Address for correspondence: Dr Michael Murray,

Discipline of Pharmacology,

University of Sydney,

NSW 2006,

AUSTRALIA

Tel: $\quad$ (61-2)-9036-3259

Fax: $\quad(61-2)-9351-4447$

email: michael.murray@sydney.edu.au 


\section{ABSTRACT.}

The multi-kinase inhibitor sorafenib (SOR) is clinically important in the treatment of hepatocellular and renal cancers and undergoes CYP3A4-dependent oxidation in liver to the pharmacologically active $\mathrm{N}$-oxide metabolite (SNO). There have been reports that kinase inhibitors such as SOR may precipitate pharmacokinetic interactions with coadministered drugs that compete for CYP3A4-mediated biotransformation, but these occur non-uniformly in patients. Clinical evidence also indicates that SNO accumulates in serum of some patients during prolonged SOR therapy. In this study undertaken in hepatic microsomes from individual donors, we assessed the possibility that SNO might contribute to pharmacokinetic interactions mediated by SOR. Enzyme kinetics of CYP3A4-mediated midazolam 1' -hydroxylation in individual human hepatic microsomes were analyzed by nonlinear regression and appropriate replots. Thus, SNO and SOR were linear-mixed inhibitors of microsomal CYP3A4 activity (Kis $15 \pm 4$ and $33 \pm 14 \mu \mathrm{M}$, respectively). To assess these findings, further molecular docking studies of SOR and SNO with the 1TQN crystal structure of CYP3A4 were undertaken. SNO elicited a larger number of interactions with key amino acid residues located in substrate recognition sequences of the enzyme. In the optimal docking pose, the N-oxide moiety of SNO was also found to interact directly with the heme moiety of CYP3A4. These findings suggest that SNO could contribute to pharmacokinetic interactions involving SOR, perhaps in individuals who produce high circulating concentrations of the metabolite.

\section{INTRODUCTION}

Multi-kinase inhibitors have significantly improved cancer treatment by selectively targeting signal transduction pathways that promote tumorigenesis. Sorafenib (SOR) is an important drug that is used in the treatment of liver and renal carcinomas (1,2; Fig. 1). Although SOR is better tolerated than conventional cytotoxic agents, a number of adverse events with these drugs, including dose-limiting toxicities such as the hand-foot syndrome and other dermal toxicities, and pharmacokinetic drugdrug interactions (DDIs) have been reported in some patients (1-4). Adverse events with SOR may require interruptions to dosage regimen or even to the termination of therapy. The pharmacokinetic behavior of SOR during therapy is complex. There is a non-linear relationship between dose and systemic exposure, and both SOR and its major activemetabolite SOR N-oxide (SNO) accumulate in serum with repeated doses (3,5-8); the extent of accumulation varies between patients. Biotransformation enzymes, especially the hepatic cytochrome P450 (CYP) mixed-function oxidases, 
are major factors that influence the rate of drug elimination. The quantitatively important human hepatic CYP3A4 mediates the oxidation of SOR to its primary metabolites, including SNO $(9,10)$. To maximize their anti-cancer actions, kinase inhibitors like SOR are being used increasingly in combination with other oncology drugs, some of which are also substrates for CYP3A4 $(3,4,8,11,12)$. Clinical studies have reported that the clearance of coadministered drugs may be impaired in some patients who receive SOR, but such interactions do not occur uniformly and are difficult to predict $(3,4,8,12)$.<smiles>CNC(=O)c1cc(Oc2ccc(NC(=O)Nc3ccc(Cl)c(C(F)(F)F)c3)cc2)ccn1</smiles><smiles>CNC(=O)c1cc(Oc2ccc(NC(=O)Nc3ccc(Cl)c(C(F)(F)F)c3)cc2)cc[n+]1[O-]</smiles>

Fig. 1. Structures of SOR and its major metabolite SNO

Personalized medicine aims to tailor the selection and dosage of drugs to the individual patient. Individuals in whom CYP3A4 oxidation is high might generate SNO more efficiently, which could lead to higher serum concentrations of the metabolite. In this study, we assessed the capacity of SOR and its major metabolite SNO to inhibit CYP3A4- mediated midazolam 1' -hydroxylation in human liver microsomes. The principal finding to emerge from kinetic studies was that SNO was $\sim 2$-fold more effective than the parent drug as an inhibitor of CYP3A4 activity. From docking studies, SNO and SOR were found to interact with key amino acid residues in the catalytic site of the enzyme that are associated with ligand binding; there was a larger number of interactions for SNO than for SOR. In addition, the N-oxide oxygen atom of SNO was in close proximity to the heme moiety in CYP3A4, consistent with its capacity to inhibit the enzyme. These findings suggest that SNO could contribute to clinically significant DDIs that have been observed in some patients during SOR therapy. 


\section{MATERIALS AND METHODS}

\section{Drugs and Chemicals}

SOR (4-[4-([4-chloro-3-(trifluoromethyl)phenyl] carbamoylamino)phenoxy]-N-methylpyridine-2carboxamide) and SNO were synthesized as described by Gillani et al. (13). Midazolam and all biochemicals were obtained from Sigma- Aldrich (Castle Hill, NSW, Australia). 1' was from Cerilliant (Round Rock, TX, USA). Microsomal fractions containing cDNA-directed CYP3A4 expressed in insect cells (Supersomes) were obtained from BD Biosciences (North Ryde, NSW, Australia). Solvents and general reagents were from LabScan (Lomb Scientific, Taren Point, NSW, Australia) or Ajax Chemicals (Sydney, NSW, Australia).

\section{Liver Donors and Preparation of Microsomal Fractions}

Experiments in human liver microsomes were approved by ethics committees of the Western Sydney Area Health Service and the University of Sydney, according to the Declaration of Helsinki. Liver segments from 16 individual donors were available for the study and were obtained through the Queensland and Australian Liver Transplant Programs (Princess Alexandria Hospital, Brisbane, Queensland, and Royal Prince Alfred Hospital, Sydney, NSW, Australia, respectively). Liver tissue was from the normal margin during orthotopic liver transplantation or resection of liver tumors and was perfused with cold Viaspan solution within 15 min of surgical removal (DuPont, Wilmington, DE, USA), and then transferred to liquid nitrogen for storage. Liver microsomes were prepared by differential ultracentrifugation (14), and protein content was quantified as described previously (15).

\section{Midazolam and SOR Biotransformation by Human Liver Microsomes}

Microsomal CYP3A-mediated midazolam 1' -hydroxylation was determined as described previously (16). Incubations $\left(37^{\circ} \mathrm{C}, 100 \mu \mathrm{g}\right.$ protein, $5 \mu \mathrm{M}$ midazolam) in $0.1 \mathrm{M}$ potassium phosphate buffer ( $\mathrm{pH}$ 7.4, $0.5 \mathrm{~mL}$ ) were initiated with NADPH $(1 \mathrm{mM})$. Linearity of product formation in incubations was established. After $5 \mathrm{~min}$, reactions were terminated (formic acid; $0.1 \% ; 1.5 \mathrm{~mL}$ ) and loaded onto SPE cartridges (Bond Elut C18; Agilent Technologies, Mulgrave, VIC, Australia). Cartridges were washed with ammonium acetate $(10 \mathrm{mM}, 1 \mathrm{~mL})$ and eluted with methanol $(2 \times 1 \mathrm{~mL})$; diazepam was the internal standard. Midazolam and its 1' $\quad$-hydroxy-metabolite were quantified by LC-MS/MS (Finnigan MAT TSQ 7000 system using electrospray ionization in the positive ion mode; San Jose, CA, USA) after separation on an Alltima C18 $5 \mu$ column (150 × 2.1 mm I.D.; HiChrome, Reading, Berks, UK). 
Microsomal SOR biotransformation was determined as described previously (10). Incubations $\left(37^{\circ} \mathrm{C}\right.$, $75 \mu \mathrm{g}$ protein, $75 \mu \mathrm{M}$ SOR) in $0.1 \mathrm{M}$ potassium phosphate buffer ( $\mathrm{pH} 7.4,0.25 \mathrm{~mL}$ ) were initiated with NADPH (1 mM); SOR was added in $2.5 \mu \mathrm{L}$ DMSO, which did not significantly affect product formation. Linearity of product formation in incubations was established. After $20 \mathrm{~min}$, reactions were terminated (acetonitrile; $0.5 \mathrm{~mL}$ ), the precipitate was removed by centrifugation, and the supernatant was dried under nitrogen; the internal standard was SOR-methyl-d3. SOR and SNO were quantified by LC-MS/MS after separation on an XTerra MS C18 $3.5 \mu$ column $(150 \times 2.1$ mm I.D.; Waters, Rydalmere, NSW, Australia).

\section{Inhibition of CYP3A4-Dependent Midazolam 1'-Hydroxylation by SOR and SNO}

Initial inhibition studies in human hepatic microsomes ( $\mathrm{n}=3$ individual livers) were undertaken at a midazolam concentration of $1 \mu \mathrm{M}$. This is within the range of total midazolam concentrations in patient plasma $(17,18)$. Total concentrations were used because this improved DDI assessments

with hydrophobic drugs (like midazolam and SOR) that may accumulate in liver (19). Kinetic studies were then conducted over a wider range of substrate concentrations (0.5-10 $\mu \mathrm{M}$ midazolam) that spanned the Km. Kinetic data were analyzed by non-linear regression of $1^{\prime}$-hydroxymidazolam formation $(\mathrm{V})$ as a function of midazolam $(\mathrm{S})$ concentration, accompanied by statistical analysis ( $\mathrm{r}$ of all regression lines; GraphPad Prism 5; San Diego, CA, USA). Data were analyzed further by Lineweaver-Burk and Dixon plots and appropriate replots to characterize the mode of inhibition (20). Ki values were obtained from the $x$-intercept of Dixon slope replots.

\section{Computational Details of the Docking of Ligands into the Active Site of CYP3A4}

X-ray crystal structures for CYP3A4 were reported in 2004 by two groups $(21,22)$, and several crystal structures are now available. The 1TQN structure of CYP3A4 was used in the present molecular docking analysis because this has been used in previous studies with SOR $(10,23)$. The CYP3A4 structure was prepared by including the unresolved residues (282-285) in the 1TQN structure, $\mathrm{H}$ atoms, and Kollman all atom charges using the Bipolymer module of SYBYL (version X-2.1, Certara, Princeton, NJ, USA). The unresolved residues in the $\mathrm{N}$ - and C-termini of the $\mathrm{X}$-ray structure, which are distant from the catalytic site, were excluded from the analysis. The three-dimensional coordinates (sdf format) of SOR and SNO and midazolam were obtained from the Pubchem server (https://pubchem.ncbi.nlm.nih.gov/). All molecular modeling was performed using SYBYL, installed on a Red Hat Linux 6.9 OS workstation. Gasteiger-Huckel partial atomic charges (24) were assigned, and 
energy minimization was done using Powell's conjugate gradient method in conjunction with a Tripos 5.2 force field $(25,26)$. A minimum energy difference of $0.001 \mathrm{kcal} / \mathrm{mol}$ was set as the convergence criterion.

Molecular docking experiments were conducted using Surflex-Dock docking suite (27). The binding site for ligand docking was defined by protomol, which identified the hemebinding site as the principal site. Molecular docking of SOR and SNO was performed in the presence and absence of midazolam. The resulting binding poses were ranked according to the total score (SYBYL). For the study of ternary complexes, two strategies were adopted. In the first approach, midazolam was docked in the CYP3A4 binding site, followed by docking of SOR or SNO. Alternately, SOR or SNO was docked in the CYP3A4 binding site, followed by the docking of midazolam.

\section{Statistics}

Data are expressed throughout as means \pm SEM of individual estimates as indicated.

Table I. Information on Demographic Factors and Drug Histories in Human Liver Donors

\begin{tabular}{|c|c|c|c|}
\hline Donor & Age & Gender & Drug history \\
\hline HL1 & 49 & F & None \\
\hline HL2 & 69 & $\mathrm{~F}$ & None \\
\hline HL5 & 48 & $\mathrm{~F}$ & None \\
\hline HL8 & 16 & $\mathrm{~F}$ & Spironolactone, ranitidine, prednisone \\
\hline HL9 & 40 & $\mathrm{~F}$ & Sucralfate \\
\hline HL10 & 35 & M & Dopamine, desmopressin \\
\hline HL11 & 46 & $\mathrm{~F}$ & $\begin{array}{l}\text { Ranitidine, epinephrine, } \\
\text { penicillin, dexamethasone }\end{array}$ \\
\hline HL12 & 6 & $\mathrm{~F}$ & Simvastatin \\
\hline
\end{tabular}

Information was not available for donors HL3, HL4, HL6, HL7, HL13-HL16 


\section{RESULTS}

Interindividual Variation in SNO Formation and Midazolam $\mathbf{1}^{\prime}$-Hydroxylation in Human Liver Microsomes

Liver tissue from 16 individual donors was available for the present study. Limited information on demographic factors and drug histories was available for eight of the donors and is shown in Table I. The age range was $6-69$, and seven of the donors were female. Two of the liver donors had received glucocorticoids, which are potential CYP3A4 inducers-prednisone (HL8) and dexamethasone (HL10) - while another donor (HL12) received the CYP3A4 substrate simvastatin; however, detailed information on dosage regimen was not available. SNO formation was measured in 14 livers (HL1HL14) and varied over a 6.5- fold range (0.06-0.39 $\mathrm{nmol} / \mathrm{mg}$ protein/min; Fig. 2). In comparison, CYP3A-mediated midazolam $1^{\prime}$-hydroxylation varied 7.8 -fold in the same fractions $(0.23-1.8$ $\mathrm{pmol} / \mathrm{mg}$ protein/min; Fig. 2).

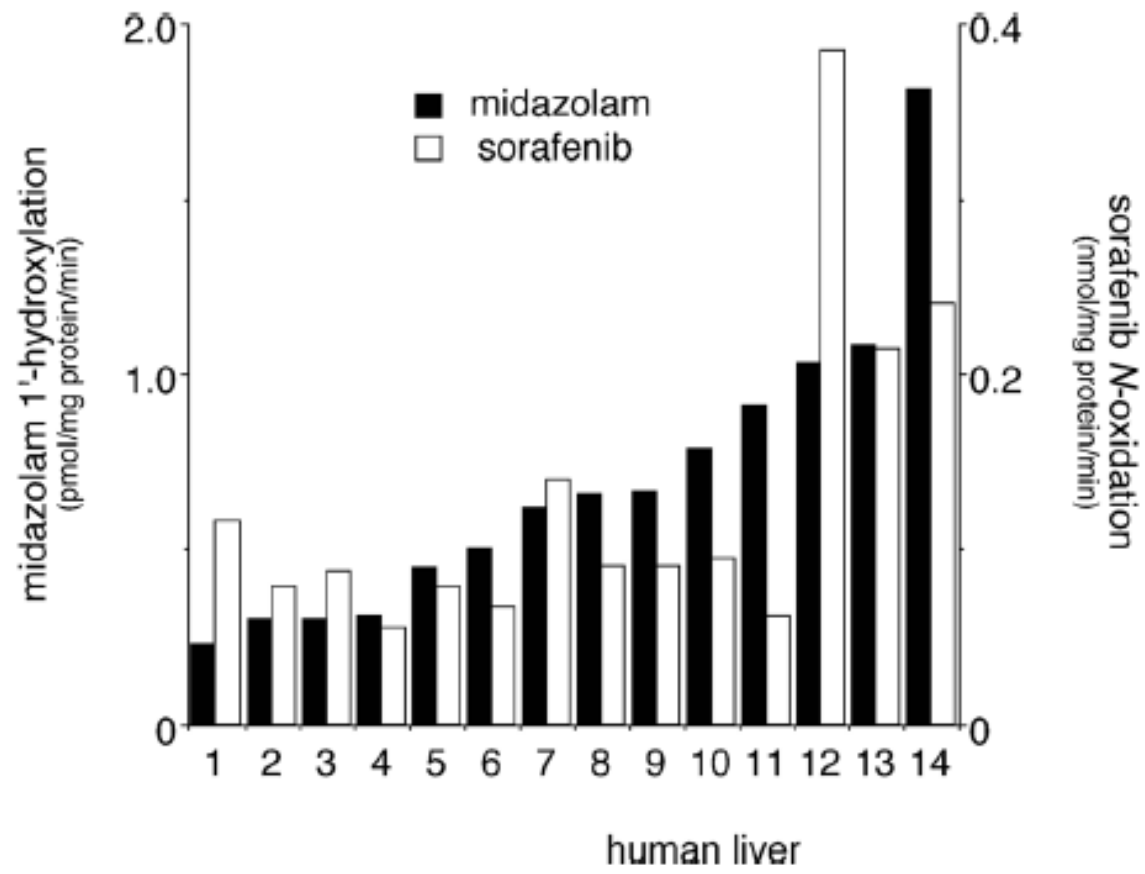

Fig. 2. Interindividual variation in midazolam $1^{\prime}$-hydroxylation and SOR N-oxidation to SNO in human liver microsomes $(n=14)$. 


\section{Comparative Inhibition of Microsomal CYP3A4-Dependent}

1'-Hydroxylation of Midazolam by SOR and Its Major Oxidized Metabolite SNO The Km for midazolam was $2.1 \pm 0.2 \mu \mathrm{M}$ in human liver microsomes, while Vmax values were in the range 50-447 pmol $1^{\prime}$ hydroxymidazolam/min/mg protein ( $\mathrm{n}=4$ livers: HL5, HL13, HL15, and HL16); these values are compatible with those reported previously $(28,29)$. In initial studies, IC50s were determined for SOR and its major metabolite SNO against the CYP3A4-catalyzed $1^{\prime}$-hydroxylation of midazolam $(1 \mu \mathrm{M})$, which is within the range of total plasma concentrations of the drug reported in clinical studies $(17,18)$. SNO exhibited an IC50 of $20 \pm 1 \mu \mathrm{M}$ ( $\mathrm{n}=3$ individual livers), whereas $50 \%$ inhibition was not attained at the highest test concentration of SOR $(50 \mu \mathrm{M})$. We corroborated these findings using cDNAexpressed human CYP3A4. IC50s of $13 \pm 1 \mu \mathrm{M}$ and $20 \pm 2 \mu \mathrm{M}$ were obtained for SNO and SOR, respectively, at a midazolam concentration of $3 \mu \mathrm{M}$. Because SNO appeared to be a more effective inhibitor than the parent drug, kinetic studies were undertaken to evaluate the interaction in greater detail. In the case of SOR, the livers HL5, HL13, and HL15 were used in experiments and HL5, HL15, and HL16 were used to evaluate SNO; representative analyses are shown in Figs. 3 and 4 . The data were fitted by alternate models of inhibition (GraphPad Prism 5). The optimal fit was obtained for linear-mixed inhibition kinetics as described by the Henri-Michaelis-Menten equation:

$$
\frac{\mathrm{V}}{\mathrm{V}_{\max }}=\frac{\mathrm{S}}{\mathrm{K}_{\mathrm{m}}\left(1+\frac{\mathrm{I}}{\mathrm{K}_{\mathrm{i}}}\right)+\mathrm{S}\left(1+\frac{\mathrm{I}}{\mathrm{K}_{\mathrm{i}}}\right)}
$$

Lineweaver-Burk and Dixon plots with appropriate replots were constructed to verify the mode of inhibition as linear-mixed (20). From the primary Lineweaver-Burk plots, the point of intersection of the lines was above the $x$-axis and to the left of the $y$-axis for both SOR and SNO (Figs. $3 b$ and $4 b$ ), while the Dixon slope replots were linear and intercepted the $x$-axis to the left of the origin (Figs. $3 e$ and $4 \mathrm{e})$. The $\mathrm{Ki}$ in the case of SNO was $15 \pm 4 \mu \mathrm{M}$, and $33 \pm 14 \mu \mathrm{M}$ for SOR. The factor $\alpha$ describes the extent to which the equilibrium constants $\mathrm{Km}$ and $\mathrm{Ki}$ are altered by inhibitor and substrate, respectively (20; Fig. 5). In the case of SNO, the value of $\alpha$ was $11 \pm 1$, while for SOR, this value was 15 \pm 6 . 

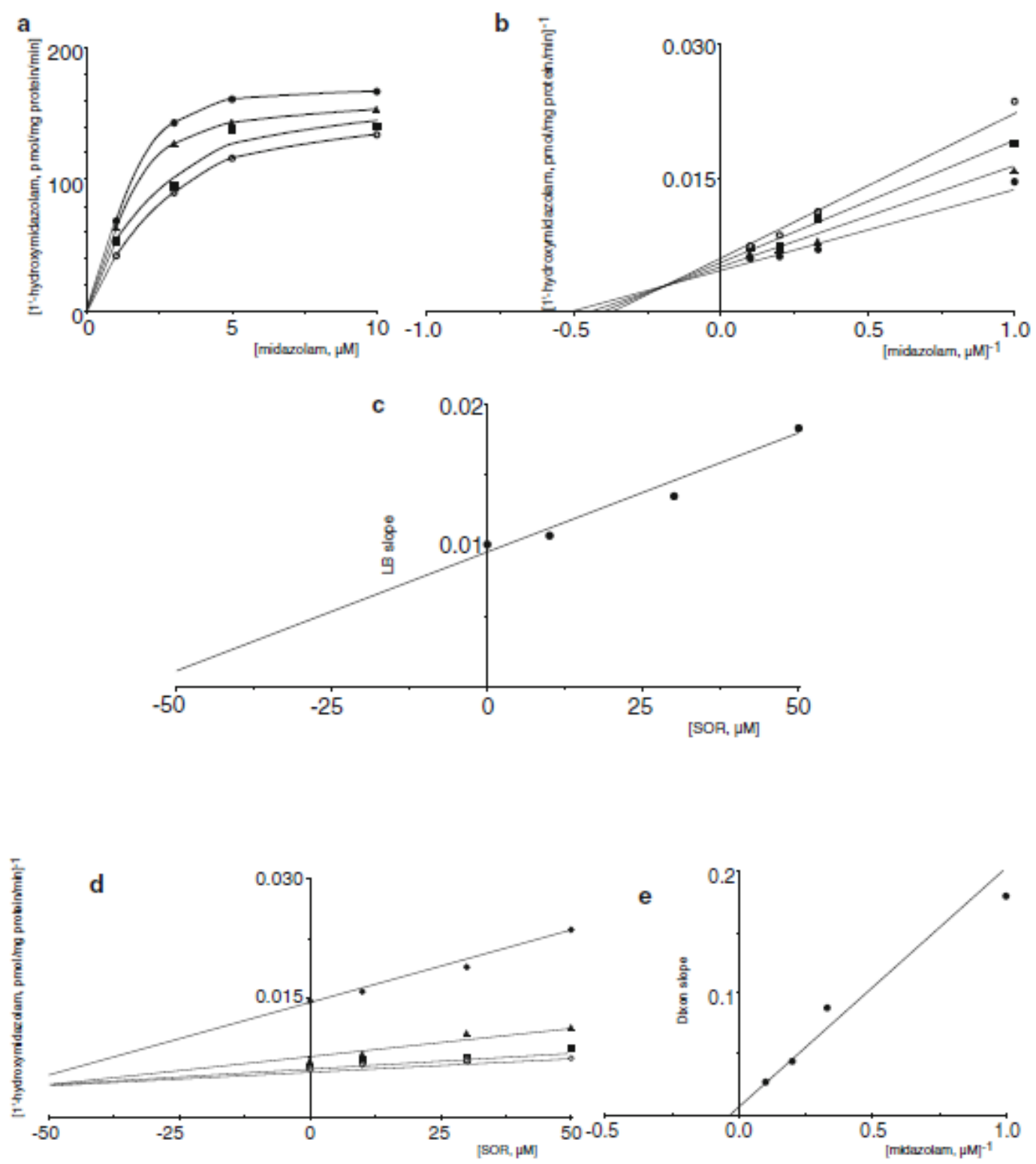

Fig. 3. Kinetic analysis of the inhibition of human microsomal CYP3A4-mediated midazolam $1^{\prime}$ hydroxylation by SOR. a Michaelis-Menten plot; key to SOR concentrations: (filled circles) $0 \mu \mathrm{M}$, (filled triangles) $10 \mu \mathrm{M}$, (filled squares) $30 \mu \mathrm{M}$, (empty circles) $50 \mu \mathrm{M}$. b Lineweaver- Burk plot. c Lineweaver-Burk slope (LB slope) replot. d Dixon plot; key to midazolam concentrations: (filled circles) $1 \mu \mathrm{M}$, (filled triangles) $3 \mu \mathrm{M}$, (filled squares) $5 \mu \mathrm{M}$, (empty circles) $10 \mu \mathrm{M}$. e Dixon slope replot. A representative analysis conducted in one of three separate microsomal fractions is shown. 

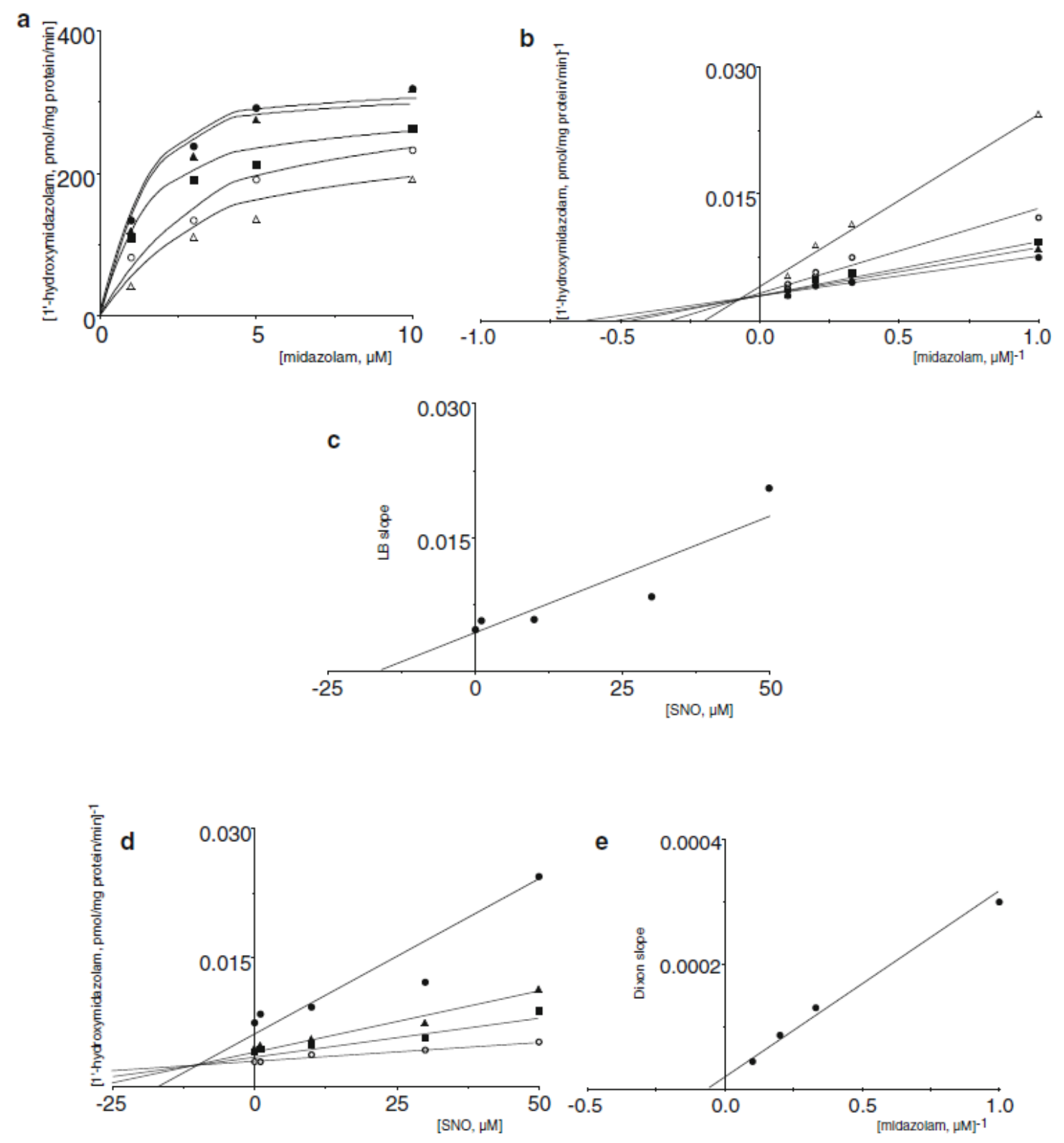

Fig. 4. Kinetic analysis of the inhibition of human microsomal CYP3A4-mediated midazolam $1^{\prime}$ hydroxylation by SNO. a Michaelis-Menten plot: key to NOX concentrations: (filled circles) $0 \mu \mathrm{M}$, (filled triangles) $1 \mu \mathrm{M}$, (filled squares) $10 \mu \mathrm{M}$, (empty circles) $30 \mu \mathrm{M}$. (empty triangles) $50 \mu \mathrm{M}$. b LineweaverBurk plot. c Lineweaver-Burk slope (LB slope) replot. d Dixon plot; key to midazolam concentrations: (filled circles) $1 \mu \mathrm{M}$, (filled triangles) $3 \mu \mathrm{M}$, (filled squares) $5 \mu \mathrm{M}$, (empty circles) $10 \mu \mathrm{M}$. e Dixon slope replot. A representative analysis conducted in one of three separate microsomal fractions is shown. 


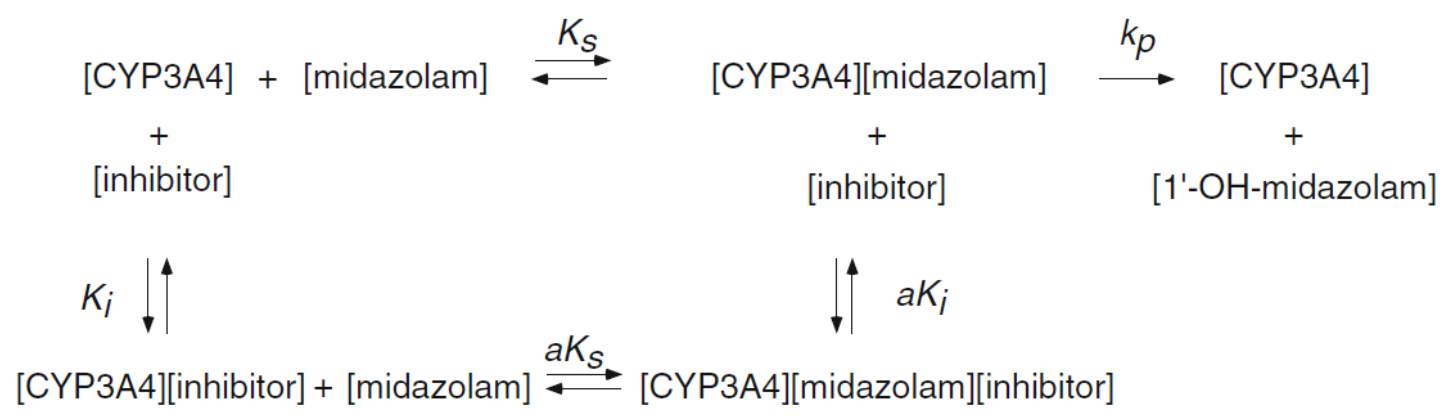

Fig. 5. Michaelis-Menten equilibria showing CYP3A4-inhibitor/substrate complex formation and linear mixed-type reversible inhibition of CYP3A4-dependent midazolam $1^{\prime}$-hydroxylation. [inhibitor] refers to either SOR or SNO (as shown in Figs. 3 and 4, respectively).

\section{Computational Modeling}

Molecular docking was undertaken to evaluate the interactions between CYP3A4 (1TQN structure) and both SOR and SNO in greater detail (Fig. 6).Docking was also performed with alternate CYP3A4 crystal structures (not shown); the binding conformations of SOR/SNO were found to be near identical using these structures. The energetically favorable binding mode of SOR showed that the distance between the atom in SOR that preferentially undergoes oxidation (the heterocyclic nitrogen atom of the pyridine ring, to form SNO) and the CYP3A4 heme iron was $3.3 \AA$ (Fig. 6a). This is compatible with the reported distance between the heme and oxidizable atom in the CYP3A4-bromoergocryptine crystal structure (30), and within the $6 \AA$ limit suggested by de Graaf et al. (31). In comparison, SNO was docked in a catalytically favourable orientation with a distance of 2.1 Å between the oxygen atom of the $\mathrm{N}$-oxide group and the heme iron (Fig. 6b). This finding is also in accord with the recent finding that SNOundergoes CYPmediated reduction at the N-oxide moiety to regenerate SOR (13).

Docking studies identified aromatic $\pi-\pi$ interactions between SOR/SNO and the residues Phe 108 and Phe 220 within the active site of CYP3A4. The structures were stabilized by further interactions in the catalytic center, including polar interactions between SOR/SNO and Asp 76, Arg 105, Arg 212, and Glu 374, and hydrophobic interactions with Ile 120, Ile 301, Ala 305, Thr 309, Ile 369, and Ala 370 . It is noteworthy that there were additional interactions between SNO and residues within the CYP3A4 catalytic site (Fig. 6b) due to differences in the binding modes for SNO and SOR (Fig. 6c). Thus, the docked pose of SNO shows direct Hbonding between the diaryl ether oxygen and Ser 119, while two of the fluorines in the aromatic CF3 group are potential H-bond acceptors for Arg 106 and Thr 224, respectively. Furthermore, halogen- $\pi$ interactions between the $\mathrm{Cl}$ atom of SNO and Phe 57 were 
observed. In addition, the aromatic residues Phe 215 and Phe 304 are involved in $\pi$-stacking interactions with the CF3-substituted aromatic system and hydrophobic interactions with the central phenyl ring of SNO, respectively. Although binding scores should be interpreted with caution (32), the total score of the docked SNO was somewhat greater than that for SOR (9.8 versus $8.2 ;$ SYBYL), which suggests that the interaction of CYP3A4 with SNO is of higher affinity than that with SOR (33).

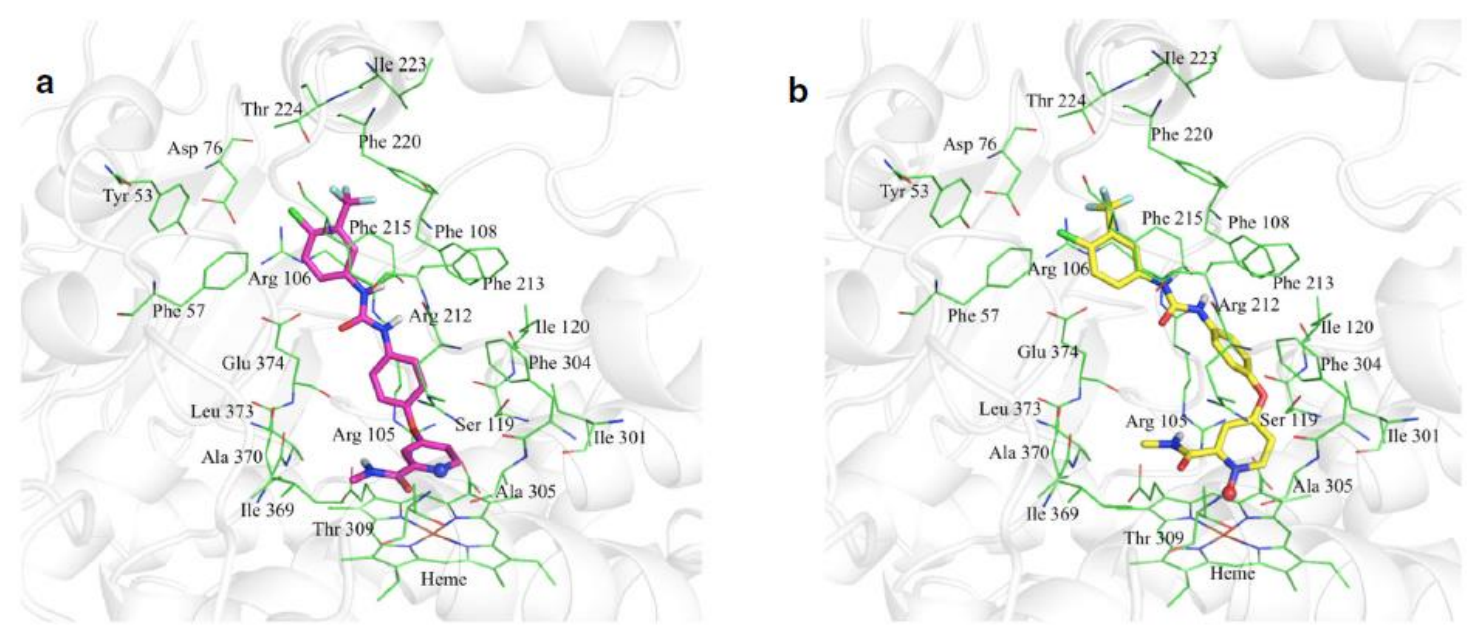

c

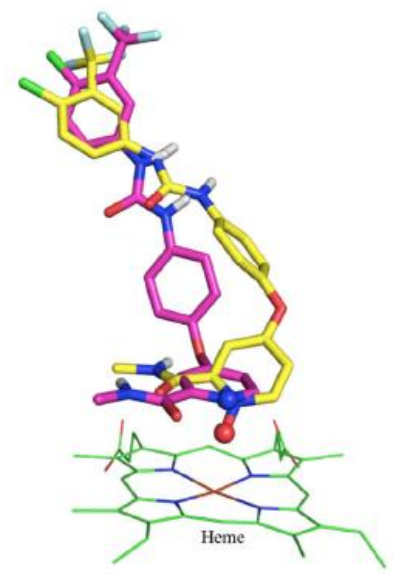

Fig. 6. Docked poses of SOR and SNO in the catalytic site of CYP3A4. a SOR, b SNO, and c overlay of SOR and SNO. Key amino acid residues involved in binding interactions are numbered

In the presence of a bound midazolam molecule, SNO and SOR were docked inefficiently; the principal site of metabolism was distant from the heme iron (>10 $\AA$; not shown). When SOR was docked within the active site of CYP3A4, midazolam was bound > $23 \AA$ from the heme. Similar findings were obtained when SNO was docked into the CYP3A4 structure when midazolam was already present.

\section{DISCUSSION}


The multi-kinase inhibitor SOR precipitates pharmacokinetic DDIs with certain coadministered drugs that are CYP3A4 substrates in some, but not all, patients who receive combination therapy. The underlying mechanism of these DDIs is unclear, but such information is important for the development of strategies that minimize DDIs in order to optimize SOR therapy. CYP3A4 is the major catalyst in the oxidation of SOR to SNO (Vmax/Km $9.4 \mathrm{pmol} / \mathrm{pmol} \mathrm{CYP} / \mathrm{h} / \mu \mathrm{M})$, whereas CYP3A5 was 13-fold less efficient (Vmax/Km $0.74 \mathrm{pmol} / \mathrm{pmol} \mathrm{CYP} / \mathrm{h} / \mu \mathrm{M} ; 10)$. The inhibitory actions of SOR toward CYP3A4mediated midazolam $1^{\prime}$-hydroxylation are reportedly enhanced in NADPH-supplemented human liver microsomes $(34,35)$. Although inhibition was timedependent, mechanism-based inhibition could not be established. In this study, we assessed the possibility that SNO-the primary oxidized and pharmacologically active metabolite of SOR-may contribute to the inhibition of CYP3A4 activity. Indeed, Giri et al. reported that another N-oxide metabolite-voriconazole N-oxide-was an effective inhibitor of CYP3A4 (IC50 $8.7 \mu \mathrm{M} ; 36$ ), and there are other CYP-mediated drug metabolites that have been shown to be more effective inhibitors than the parent drugs. These include not only reactive metabolites generated by CYP oxidation of alkylamino, methylenedioxyphenyl, thionosulfur, phenolic, and unsaturated moieties (37-43) but also major isolable metabolites that elicit reversible inhibition of CYPs (44-46).

From kinetic studies, the Ki for SNO against midazolam $1^{\prime}$-hydroxylation was $15 \pm 4 \mu \mathrm{M}$ and the Km for midazolam was $\sim 2 \mu \mathrm{M}$. Therefore, CYP3A4 has an $\sim 7.5$-fold greater affinity for midazolam than for SNO. In comparison, the higher Ki value for SOR ( $33 \pm 14 \mu \mathrm{M})$ indicates that the affinity of CYP3A4 for midazolam is $\sim 16$-fold that of SOR. Whereas SOR has been found previously to be a moderate inhibitor of the enzyme (23), to our knowledge, the metabolite SNO has not been evaluated in detail as a CYP3A4 inhibitor. The present findings suggest that SNO is a major metabolite of SOR that mediates reversible CYP3A4 inhibition.

The pharmacokinetics of SOR are complex and exhibit extensive interindividual variation $(5,6)$. The area under the serum concentration versus time curve (AUC) for SOR, which reflects systemic drug exposure, was increased in patients who received prolonged therapy with the drug (4-6). This could reflect impaired CYP-dependent clearance by metabolites such as SNO that has also been shown to accumulate in serum in a number of patients $(7,8)$. Thus, SOR and SNO accumulated several fold in three of eight patients after 12 days of SOR treatment, compared with maximal serum concentrations at day 7 (7). Indeed, the maximal serum concentration ( $\mathrm{Cmax}$ ) of SNO reached $8.5 \mu \mathrm{M}$ in one patient, 
which is close to the Ki for CYP3A4 inhibition that was estimated in the present study. Several other studies also reported variable SNO pharmacokinetics in patient serum over the approximate range 1$5 \mu \mathrm{M}(4,6,8,47,48)$. SOR has been used increasingly in combination with other anticancer agents, but these have the potential to elicit pharmacokinetic DDIs and increase the likelihood of adverse effects. The combination of doxorubicin $(60 \mathrm{mg} / \mathrm{m} 2)$ and SORat several dose levels was generally well tolerated, but some patients who received the highest dose of SOR exhibited significant increases in the Cmax and AUC of doxorubicin (11). Interpatient variation in these parameters was pronounced. More recently, the AUCO-24 for docetaxel $(100 \mathrm{mg} / \mathrm{m} 2)$ was found to be increased on average by $54 \%$ following administration of $200 \mathrm{mg}$ SOR twice daily, and up to $80 \%$ at the standard therapeutic dose of $400 \mathrm{mg}$ twice daily(3). These authors also reported that SOR inhibited docetaxel hydroxylation in microsomes with an IC50 of $\sim 17 \mu \mathrm{M}$. Similarly, the Cmax of vinorelbine was increased to 1.6-fold of control in patients with metastatic breast cancer who were also administered SOR (8). Further, in some colorectal cancer patients who received the SOR/irinotecan combination, exposures to irinotecan and its active metabolite SN38 were increased over those with irinotecan alone, suggesting that SOR and/or SNO might inhibit irinotecan biotransformation that is mediated in part by CYP3A4 $(4,12)$. These studies all recommended caution in the management of patients receiving combinations of SOR and oncology drugs that require CYP3A4 for biotransformation.

SNO and SOR were linear mixed-type inhibitors of microsomal midazolam $1^{\prime}$-hydroxylation. The parameter $\alpha$ indicates the extent to which inhibitor and substrate decrease the apparent affinity of the enzyme for substrate and inhibitor, respectively (20). In the present study, the values of $\alpha$ for SNO and SOR were in the range $11-15$, indicating that these molecules significantly decrease the capacity of CYP3A4 to interact with midazolam. The active site of unliganded CYP3A4 has a large volume ( 950 $\AA 33$; 21). However, the active site also appears to have multiple binding pockets that undergo significant conformational changes on binding of substrates; this accounts for structural diversity and cooperative interactions between substrates (49-53). To evaluate the present kinetic findings further, we undertook molecular modeling of the SOR/SNO-CYP3A4 interactions using the 1TN crystal structure that has been used previously to analyze SOR binding $(10,23)$. Docking studies with CYP3A4 identified aromatic $\pi-\pi$ interactions between both SOR and SNO and the residues Phe 108 and Phe 220, polar interactions with Asp 76, Arg 105, Arg 212, and Glu 374, and hydrophobic interactions with Ile 120, lle 301, Ala 305, Thr 309, lle 369, and Ala 370. These hydrophobic residues were found previously to be important in midazolam binding (54). Figure 7 shows the location of the residues in CYP3A4 that were involved in interactions with SOR and SNO in relation to the protein helical domains 
and $\beta$-sheets, and also the substrate recognition sequences (SRS) that comprise the active center of the enzyme (55). Amino acids that interacted with both SOR and SNO were located in SRS-1 (which spans residues 101-123), SRS-2 (which spans 204-216), SRS-4 (which spans 294-312), and SRS-5 (368376), but not SRS-3 or SRS-6. In SRS-1, three residues were involved in SOR/ SNO interactions (Phe 108, lle 120, and Arg 105), one was located in SRS-2 (Arg 212), three were in SRS-4 (Ala 305, lle 301, and Thr 309), and three were in SRS-5 (Glu 374, Ile 369, and Ala 370). Phe 220 and Arg 76 were also involved in SOR/ SNO interactions but were outside SRS regions.

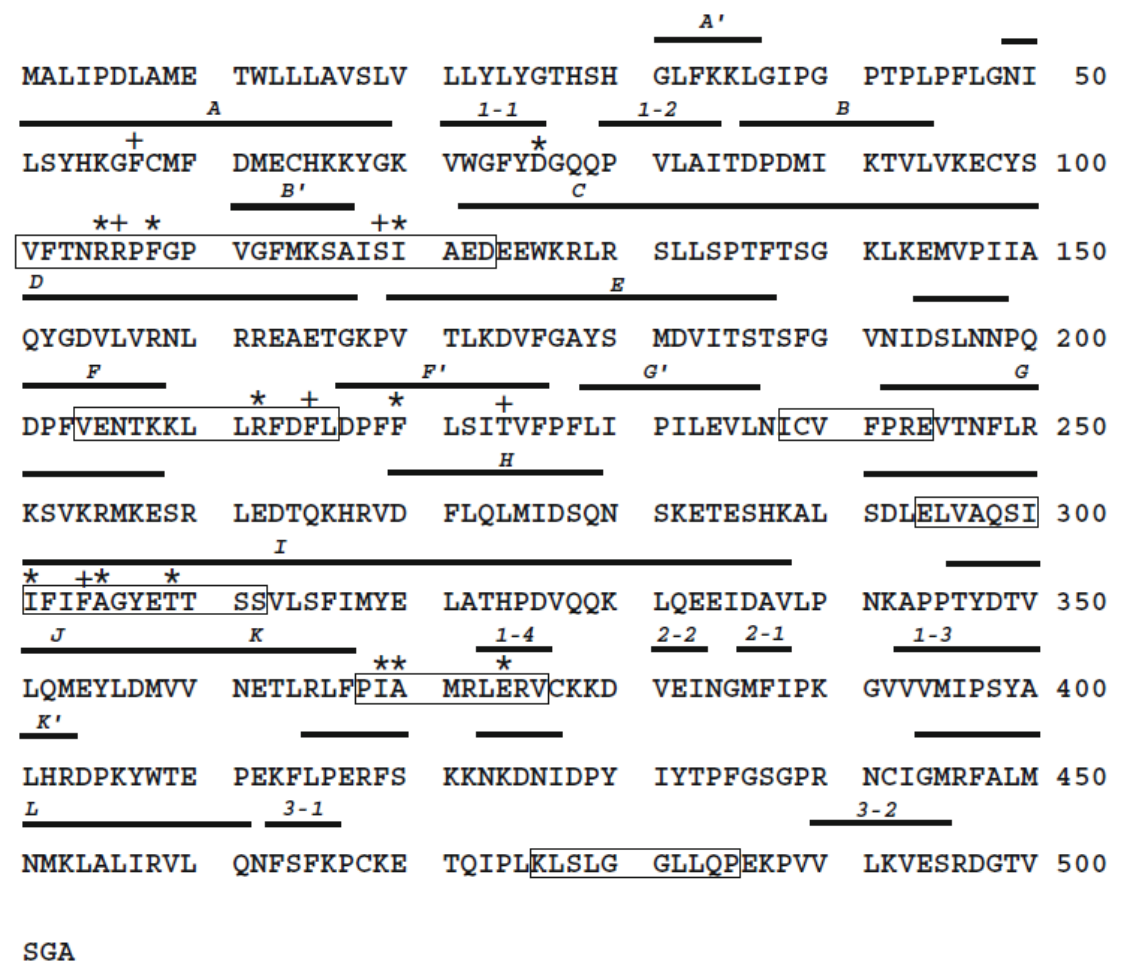

Fig. 7. Amino acid sequence of CYP3A4 showing the location of residues implicated in binding interactions with both SOR and SNO (asterisks) and SNO alone (crosses). Bars indicate the locations of helical domains (italicized letters) and $\beta$-sheets (italicized numbers). Amino acid residues in SRS regions are shown in boxes.

From kinetic studies, the interaction of CYP3A4 with SNO was found to be of higher affinity than that with SOR. In accord with these findings, additional binding interactions emerged from the SNOCYP3A4 docking analysis, including H-bonding with Ser 119, $\operatorname{Arg} 106$, and $\operatorname{Thr} 224$, halogen- $\pi$ interactions between the $\mathrm{Cl}$ atom of SNO and Phe 57, a $\pi$-stacking interaction between the 
trifluorophenyl ring and Phe 215, and hydrophobic interactions with Phe 304. Two of these additional interactions involved residues in SRS-1 (Ser 119 and Arg 106), one involved the SRS-2 residue Phe 215 and one involved the SRS-4 residue Phe 304. The non-SRS residues Phe 57 and Thr 224 also contributed to the SNO-CYP3A4 interaction. Previous studies identified roles for several of these additional residues in the interaction ofCYP3A4 with other ligands. Thus, Ser 119 was associated with Hbonding/polar interactions with ritonavir analogues (56), aflatoxin B1 (51), and ketoconazole (52); Arg 212 and Thr 224 were important for the orientation of bromoergocryptine in the CYP3A4 active site (30) and Phe 304 was also involved in aflatoxin B1 binding (52). Like SNO, these ligands are effective CYP3A4 inhibitors and/or substrates.

The present docking analysis also offers an explanation to account for the recently reported NADPHdependent aerobic reduction of SNO to SOR that is mediated by human CYPs (13). The mechanism of this reaction has been proposed to involve direct interaction of the N-oxygen atom in SNO with the ferrous CYP heme iron (13). It is now apparent that the heme $\mathrm{N}$-oxide oxygen interaction is stabilized by multiple interactions with amino acid residues in SRS regions that are located within the active center of CYP3A4 (Fig. 6b). Subsequent reduction by an electron from NADPH delivered via the NADPH-CYP reductase could then facilitate nitrogen-oxygen bond cleavage in SNO, with transfer of the oxygen atom to the heme iron, and liberation of SOR and water; similar reactions have been reported for certain other drug and xenobiotic $\mathrm{N}$-oxides $(57,58)$.

Taken together, the present findings suggest that interindividual differences in SNO formation and its accumulation in serum could contribute to variableDDIs in patients who receive SOR. Additional evidence for the capacity of SNO to elicit in vivo DDIs with SOR could be obtained using SOR-treated CYP3A4-transgenic mice (59), which could enable increased concentrations of the metabolite in serum to be generated provided an appropriate dosage regimen is used. Such studies could complement aspects of the present study. However, it would now be of interest to assess SNO production directly in patients who are receiving SOR. Indeed, monitoring of serum SNO concentrations over time may enable individuals to be identified whomight benefit fromdrug regimenmodifications in order to avoid SOR-mediated DDIs.

\section{CONCLUSIONS}

The multi-kinase inhibitor SOR precipitates pharmacokinetic DDIs in some, but not all, patients. In the present study, the major SOR metabolite SNO was found to be a more potent mixed-type inhibitor of CYP3A4 than the parent drug. Docking studies identified a number of binding interactions between 
SOR and SNO and amino acid residues in the active site of the enzyme. The larger number of interactions in the docking of SNO, as well as the direct ligation of the N-oxide moiety to the CYP heme iron, accounts for its greater inhibitory potency toward CYP3A4. This finding also provides a potential explanation for the recent report that SNOundergoes CYP-mediated reduction back to SOR. The overproduction of the inhibitory metabolite SNO, and its accumulation in serum of some patients, could contribute to the interindividual variation in pharmacokinetic DDIs involving SOR.

\section{ACKNOWLEDGEMENTS}

SC acknowledges Fellowship support from the Swiss National Science Foundation. PCN acknowledges the Flinders Centre for Innovation in Cancer (FCIC) and Flinders Medical Centre (FMC) Foundation for an Early Career Research Grant. Technical contributions in aspects of the study from Dr. S. Cui, Ms. K Bourget, Mr. E Li and Ms. L Pham are also acknowledged. The supply of several of the human liver samples used in this study by Dr. J George is also gratefully acknowledged.

\section{FUNDING INFORMATION}

This study is financially supported by the Cancer Council NSW (grants RG09-14 and IG11-33).

\section{COMPLIANCE WITH ETHICAL STANDARDS}

Experiments in human liver microsomes were approved by ethics committees of the Western Sydney Area Health Service and the University of Sydney, according to the Declaration of Helsinki. Conflicts of Interest The authors declare that they have no conflict of interest.

\section{REFERENCES}

1. Escudier B, Eisen T, Stadler WM, Szczylik C, Oudard S, Siebels M, et al. Sorafenib in advanced clearcell renal-cell carcinoma. New Engl J Med. 2007;356:125-34. https://doi.org/10.1056/ NEJMoa060655.

2. Liu L, Cao Y, Chen C, Zhang X, McNabola A, Wilkie D, et al. Sorafenib blocks the RAF/MEK/ERK pathway, inhibits tumor angiogenesis, and induces tumor cell apoptosis in hepatocellular carcinoma model PLC/PRF/5. Cancer Res. 2006;66:11851-8. https://doi.org/10.1158/0008-5472.CAN-06-1377.

3. Awada A, Hendlisz A, Christensen O, Lathia CD, Bartholomeus S, Lebrun F, et al. Phase I trial to investigate the safety, pharmacokinetics and efficacy of sorafenib combined with docetaxel in patients with advanced refractory solid tumours. Eur J Cancer. 2012;48:465-74. https://doi.org/10.1016/ j.ejca.2011.12.026. 
4. Azad N, Dasari A, Arcaroli J, Taylor GE, Laheru DA, Carducci MA, et al. Phase I pharmacokinetic and pharmacodynamics study of cetuximab, irinotecan and sorafenib in advanced colorectal cancer. Investig New Drugs. 2013;31:345-54. https:// doi.org/10.1007/s10637-012-9820-z.

5. Strumberg D, Clark JW, Awada A, Moore MJ, Richly H, Hendlisz A, et al. Safety, pharmacokinetics, and preliminary antitumor activity of sorafenib: a review of four phase I trials in patients with advanced refractory solid tumors. Oncologist. 2007;12:426-37. https://doi.org/10.1634/theoncologist.12-4-426.

6. Minami H, Kawada K, Ebi H, Kitagawa K, Kim Yl, Araki K, et al. Phase I and pharmacokinetic study of sorafenib, an oral multikinase inhibitor, in Japanese patients with advanced refractory solid tumors. Cancer Sci. 2008;99:1492-8. https:// doi.org/10.1111/j.1349-7006.2008.00837.x.

7. Inaba H, Rubnitz JE, Coustan-Smith E, Li L, Furmanski BD, Mascara GP, et al. Phase I pharmacokinetic and pharmacodynamics study of the multikinase inhibitor sorafenib in combination with clofarabine and cytarabine in pediatric relapsed/ refractory leukemia. J Clin Oncol. 2011;29:3293-300. https:// doi.org/10.1200/JCO.2011.34.7427.

8 Ferrario C, Strepponi I, Esfahani K, Charamis H, Langleben A, Scarpi E, et al. Phase I/II trial of sorafenib in combination with vinorelbine as first-line chemotherapy for metastatic breast cancer. PLoS One. 2016;11:e0167906. https://doi.org/10.1371/ journal.pone.0167906.

9. Lathia C, Lettieri J, Cihon F, Gallentine M, Radtke M, Sundaresan P. Lack of effect of ketoconazolemediated CYP3A inhibition on sorafenib clinical pharmacokinetics. Cancer Chemother Pharmacol. 2006;57:685-92. https://doi.org/10.1007/ s00280-005-0068-6.

10. Ghassabian S, Rawling T, Zhou F, Doddareddy MR, Tattam BN, Hibbs DE, et al. Role of human CYP3A4 in the biotransformation of sorafenib to its major oxidized metabolites. Biochem Pharmacol. 2012;84:215-23. https://doi.org/10.1016/j.bcp.2012.04.001.

11. Richly H, Henning BF, Kupsch P, Passarge K, Grubert M, Hilger RA, et al. Results of a phase I trial of sorafenib (BAY 43- 9006) in combination with doxorubicin in patients with refractory solid tumors. Ann Oncol. 2006;17:866-73. https://doi.org/ 10.1093/annonc/mdl017.

12. Mross K, Steinbild S, Baas F, Gmehling D, Radtke M, Voliotis D, et al. Results from an in vitro and a clinical/pharmacological phase I study with the combination irinotecan and sorafenib. Eur J Cancer. 
2007;43:55-63. https://doi.org/10.1016/j.ejca.2006.08.032. 13. Gillani T, Rawling T, Murray M. Cytochrome P450-mediated biotransformation of sorafenib and its $\mathrm{N}$-oxide metabolite: implications for cell viability and human toxicity. Chem Res Toxicol. 2015;28:92-102. https://doi.org/10.1021/tx500373g.

14. Farrell GC, Koltai A, Murray M. The source of raised estrogens in male rats with portal bypass. J Clin Invest. 1988;81:221-8. https://doi.org/10.1172/JCl113299.

15. Murray M. Participation of a cytochrome P450 enzyme from the $2 \mathrm{C}$ subfamily in progesterone 21 hydroxylation in sheep liver. J Steroid Biochem Mol Biol. 1992;43:591-3. https://doi.org/ 10.1016/0960-0760(92)90248-H.

16. Ghassabian S, Chetty M, Tattam BN, Glen J, Rahme J, Stankovic Z, et al. A high-throughput assay using liquidchromatography/tandem mass spectrometry for simultaneous in vivo phenotyping of five major cytochrome P450 enzymes in patients. Ther Drug Monit. 2009;31:239-46. https://doi.org/ 10.1097/FTD.0b013e318197e1bf.

17. Smith MT, Eadie MJ, Brophy TO. The pharmacokinetics of midazolam in man. Eur J Clin Pharmacol. 1981;19:271-8. https:// doi.org/10.1007/BF00562804. 18. Crevoisier C, Ziegler WH, Eckert M, Heizmann P. Relationship between plasma concentration and effect of midazolam after oral61S. https://doi.org/10.1111/j.1365-2125.1983.tb02271.x.

19. von Moltke LL, Greenblatt DJ, Cotreau-Bibbo MM, Duan SX, Harmatz JS, Shader RI. Inhibition of desipramine hydroxylation in vitro by serotonin-reuptake-inhibitor antidepressants, and by uinidine and ketoconazole: a model system to predict drug interactions in vivo. J Pharmacol Exp Ther. 1994;268:1278-83.

20. Murray M. Metabolite intermediate complexation of microsomal cytochrome P450 2C11 in male rat liver by nortriptyline. Mol Pharmacol. 1992;42:931-8. 21. Yano JK, Wester MR, Schoch GA, Griffin $\mathrm{KJ}$, Stout CD, Johnson EF. The structure of human microsomal cytochrome P450 3A4 determined by Xray crystallography to 2.05-Å resolution. J Biol Chem. 2004;279:38091-4. https://doi.org/ 10.1074/jbc.C400293200. 
22. Williams PA, Cosme J, Vinkovic DM, Ward A, Angove HC, Day PJ, et al. Crystal structures of human cytochrome P450 3A4 bound to metyrapone and progesterone. Science. 2004;305:683- 6 . https://doi.org/10.1126/science.1099736.

23. Sugiyama M, Fujita K, Murayama N, Akiyama Y, Yamazaki H, Sasaki Y. Sorafenib and sunitinib, two anticancer drugs, inhibit CYP3A4-mediated and activate CY3A5-mediated midazolam $1^{\prime}$ hydroxylation. Drug Metab Dispos. 2011;39:757-62. https:// doi.org/10.1124/dmd.110.037853.

24. Gasteiger J, Marsili M. Iterative partial equalization of orbital electronegativity-a rapid access to atomic charges. Tetrahedron. 1980;36:3219-28. https://doi.org/10.1016/0040- 4020(80)80168-2.

25. Powell MJD. Restart procedures for the conjugate gradient method. Math Program. 1977;12:24154. https://doi.org/10.1007/ BF01593790.

26. Clark M, Cramer RD III, Van Opdenbosch N. Validation of the general purpose tripos 5.2 force field. J Comput Chem. 1989;10:982-1012. https://doi.org/10.1002/jcc.540100804.

27. Jain AN. Surflex: fully automatic flexible molecular docking using a molecular similarity-based search engine. J Med Chem. 2003;46:499-511. https://doi.org/10.1021/jm020406h.

28. Ghosal A, Satoh H, Thomas PE, Bush E, Moore D. Inhibition and kinetics of cytochrome P4503A activity in microsomes from rat, human, and cDNA-expressed human cytochrome P450.

Drug Metab Dispos. 1996;24 940-7.

29. Patki KC, Von Moltke LL, Greenblatt DJ. In vitro metabolism of midazolam, triazolam, nifedipine, and testosterone by human liver microsomes and recombinant cytochromes P450: role of CYP3A4 and CYP3A5. Drug Metab Dispos. 2003;31:938-44. https://doi.org/10.1124/dmd.31.7.938.

30. Sevrioukova IF, Poulos TL. Structural and mechanistic insights into the interaction of cytochrome P4503A4 with bromoergocryptine, a type I ligand. J Biol Chem. 2012;287:3510-7. https://doi.org/10.1074/jbc.M111.317081.

31. de Graaf C, Oostenbrink C, Keizers PH, van Vugt-Lussenburg BM, van Waterschoot RA, TschirretGuth RA, et al. Molecular modeling-guided site-directed mutagenesis of cytochrome P450 2D6. Curr Drug Metab. 2007;8:59-77. https://doi.org/10.2174/ 138920007779315062. 
32. Ferrara P, Gohlke H, Price DJ, Klebe G, Brooks CL 3rd. Assessing scoring functions for protein-ligand interactions. J Med Chem. 2004;47:3032-47. https://doi.org/10.1021/jm030489h.

33. Pham TA, Jain AN. Parameter estimation for scoring proteinligand interactions using negative training data. J Med Chem. 2006;49:5856-68. https://doi.org/10.1021/jm050040j.

34. Kenny JR, Mukadam S, Zhang C, Tay S, Collins C, Galetin A, et al. Drug-drug interaction potential of marketed oncology drugs: in vitro assessment of time-dependent cytochrome P450 inhibition, reactive metabolite formation and drug-drug interaction prediction. Pharm Res. 2012;29:1960-76. https://doi.org/10.1007/s11095-012-0724-6.

35. Filppula AM, Neuvonen PJ, Backman JT. In vitro assessment of time-dependent inhibitory effects on CYP2C8 and CYP3A activity by fourteen protein kinase inhibitors. Drug Metab Dispos. 2014;42:1202-9. https://doi.org/10.1124/dmd.114.057695.

36. Giri P, Naidu S, Patel N, Patel H, Srinivas NR. Evaluation of in vitro cytochrome P450 inhibition and in vitro fate of structurally diverse $\mathrm{N}$-oxide metabolites: case studies with clozapine, levofloxacin, roflumilast, voriconazole and zopiclone. Eur J Drug Metab Pharmacokinet. 2017;42:677-88. https:// doi.org/10.1007/s13318-016-0385-7.

37. Marcus CB, Murray M, Wilkinson CF. Spectral and inhibitory interactions of methylenedioxyphenyl and related compounds with purified isozymes of cytochrome P-450. Xenobiotica. 1985;15:351-62. https://doi.org/10.3109/00498258509045370.

38. Butler AM, Murray M. Inhibition and inactivation of constitutive cytochromes P450 in rat liver by parathion. Mol Pharmacol. 1993;43:902-8.

39. Ortiz de Montellano PR, Correia MA. Suicidal destruction of cytochrome P-450 during oxidative drug metabolism. Annu Rev Pharmacol Toxicol. 1983;23:481-503. https://doi.org/10.1146/ annurev.pa.23.040183.002405.

40. He K, Iyer KR, Hayes RN, Sinz MW, Woolf T, Hollenberg PF. Inactivation of cytochrome P450 3A4 by bergamottin, a component of grapefruit juice. Chem Res Toxicol. 1998;11:252-9. https://doi.org/10.1021/tx970192k. 
41. Stupans I, Murray M, Kirlich A, Tuck K, Hayball P. Inactivation of cytochrome P450 by the foodderived complex phenol oleuropein. Food Chem Toxicol. 2001;39:1119-24. https:// doi.org/10.1016/S0278-6915(01)00060-6.

42. Murray M. Complexation of cytochrome P-450 isoenzymes in hepatic microsomes from SKF 525A-induced rats. Arch Biochem Biophys. 1988;262:381-8. https://doi.org/10.1016/00039861(88)90202-0.

43. Murray M, Field SL. Inhibition and metabolite complexation of rat hepatic microsomal cytochrome P450 by tricyclic antidepressants. Biochem Pharmacol. 1992;43:2065-71. https://doi.org/ 10.1016/0006-2952(92)90163-D.

44. Murray M. In vitro effects of quinoline derivatives on cytochrome P-450 and aminopyrine Ndemethylase activity in rat hepatic microsomes. Biochem Pharmacol. 1984;33:3277-81. https://doi.org/10.1016/0006-2952(84)90090-X.

45. Nemeroff CB, DeVane CL, Pollock BG. Newer antidepressants and the cytochrome P450 system. Am J Psychiatr. 1996;153:311- 20. https://doi.org/10.1176/ajp.153.3.311.

46. Ohyama K, Nakajima M, Suzuki M, Shimada N, Yamazaki H, Yokoi T. Inhibitory effects of amiodarone and its $\mathrm{N}$-deethylated metabolite on human cytochrome P450 activities: prediction of in vivo drug interactions. $\mathrm{Br} J$ Clin Pharmacol. 2000;49:244-53. https://doi.org/10.1046/j.13652125.2000.00134.x.

47. Miller AA, Murry DJ, Owzar K, Hollis DR, Kennedy EB, Abou-Alfa G, et al. Phase I and pharmacokinetic study of sorafenib in patients with hepatic or renal dysfunction: CALGB 60301. J Clin Oncol. 2009;27:1800-5. https://doi.org/10.1200/JCO.2008.20.0931.

48. Flaherty KT, Lathia C, Frye RF, Schuchter L, Redlinger M, Rosen M, et al. Interaction of sorafenib and cytochrome P450 isoenzymes in patients with advanced melanoma: a phase I/II pharmacokinetic interaction study. Cancer Chemother Pharmacol. 2011;68:1111-8.https://doi.org/10.1007/s00280$\underline{011-1585-0 .}$

49. Ekroos M, Sjögren T. Structural basis for ligand promiscuity in cytochrome P450 3A4. Proc Natl Acad Sci U S A. 2006;103:13682-7. https://doi.org/10.1073/pnas.0603236103. 50. Teixeira VH, Ribeiro 
V, Martel PJ. Analysis of binding modes of ligands to multiple conformations of CYP3A4. Biochim Biophys Acta. 2010;1804:2036-45. https://doi.org/10.1016/ j.bbapap.2010.06.008.

51. Bren U, Oostenbrink C. Cytochrome P450 3A4 inhibition by ketoconazole: tackling the problem of ligand cooperativity using molecular dynamics simulations and free-energy calculations. J Chem Inf Model. 2012;52:1573-82. https://doi.org/10.1021/ ci300118x.

52. Bren U, Fuchs JE, Oostenbrink C. Cooperative binding of aflatoxin B1 by cytochrome P450 3A4: a computational study. Chem Res Toxicol. 2014;27:2136-47. https://doi.org/10.1021/ tx5004062.

53. Nair PC, McKinnon RA, Miners JO. Cytochrome P450 structure-function: insights from molecular dynamics simulations. Drug Metab Rev. 2016;48:434-52. https://doi.org/10.1080/ 03602532.2016.1178771.

54. Sevrioukova IF, Poulos TL. Structural basis for regiospecific midazolam oxidation by human cytochrome P450 3A4. Proc Natl Acad Sci U S A. 2017;114:486-91. https://doi.org/10.1073/ pnas.1616198114.

55. Torimoto N, Ishii I, Toyama KI, Hata M, Tanaka K, Shimomura H, et al. Helices F-G are important for the substrate specificities of CYP3A7. Drug Metab Dispos. 2007;35:484-92. https:// doi.org/10.1124/dmd.106.011304. 56. Kaur P, Chamberlin AR, Poulos TL, Sevrioukova IF. Structurebased inhibitor design for evaluation of a CYP3A4 pharmacophore model. J Med Chem. 2016;59:4210-20. https:// doi.org/10.1021/acs.jmedchem.5b01146.

57. Seto Y, Guengerich FP. Partitioning between N-dealkylation and N-oxygenation in the oxidation of N,N-dialkylarylamines catalyzed by cytochrome P450 2B1. J Biol Chem. 1993;268:9986-97.

58. Skálová L, Nobilis M, Szotáková B, Wsól V, Kvasnicková E. Induction and inhibition of cytochrome P450-catalysed reduction of biologically active benfluron N-oxide. Drug Metabol Drug Interact. 1998;14:221-33. https://doi.org/10.1515/ DMDI.1998.14.4.221.

59. Ly JQ, Messick K, Qin A, Takahashi RH, Choo EF. Utility of CYP3A4 and PXR-CAR-CYP3A4/3A7 transgenic mouse models to assess the magnitude of CYP3A4 mediated drugdrug interactions. Mol Pharm. 2017;14:1754-9. https://doi.org/ 10.1021/acs.molpharmaceut.7b00006. 